Cite this: RSC Adv., 2019, 9, 34754

Received 10th August 2019

Accepted 24th September 2019

DOI: $10.1039 / c 9 r a 06237 h$

rsc.li/rsc-advances
Check for updates

\section{Fabrication and characterization of a novel corn straw/modified ammonium lignosulfonate bio- composite strengthened by polyethylenimine pretreatment}

\begin{abstract}
Sidan Li, (D) $\dagger^{\mathrm{ab}}$ Yuan Yuan, (D) $\dagger^{\mathrm{c}}$ Jinman Wang ${ }^{\mathrm{a}}$ and Minghui Guo (D) *a
This study focuses on the development of novel bio-composites via the pretreatment of corn straw particles (PCSP) and modified ammonium lignosulfonate (MAL) as a binder. The corn stalk particles (CSP) were pretreated with polyethylenimine (PEI) to enhance compatibility. The effects of PEI dosage on the mechanical properties and dimensional stability were examined, where PEI pretreatment improved the interfacial properties of MAL and CSP considerably. The optimum values of the PCSP/MAL composites complied with the Chinese national standard for load-bearing particleboard. Furthermore, the results confirmed that PEI pretreatment resulted in good surface activity and exhibited a favorable effect on the crystallinity of the PCSP/MAL composites. The storage moduli $E^{\prime}$ and $\tan \delta$ peak of the PCSP/MAL composites were considerably greater than those of the CSP/MAL composites. The fractured morphology of the composites clearly showed that PEI pretreatment improves the interfacial adhesion of MAL and CSP.
\end{abstract}

\section{Introduction}

The overwhelming depletion of our natural forests has spurred governments into proclaiming limits on the exploitation of wood sources. Hence, it has become increasingly important to manufacture board from non-wood sources. Different types of bindless boards from non-wood plant fiber sources have been developed, which include wheat straw, ${ }^{1}$ corn stalk, ${ }^{2,3}$ kenaf straw $^{4-6}$ and rice straw. ${ }^{7}$ Zhou et al. ${ }^{8}$ developed an environmentally friendly thermal insulation material from cotton stalk fibers eliminating the need for resins and chemical additives. However, binderless boards have poor bond properties, mildew resistance and water resistance. ${ }^{9}$ These deficiencies limit their industrial applications, ${ }^{\mathbf{1 0}}$ hence, various types of adhesives are employed in non-wood plant boards, such as UF resin, ${ }^{11,12}$ PF resin, ${ }^{\mathbf{1 3}}$ MUF thermoset resin, ${ }^{\mathbf{1 4}}$ and polymeric diphenylmethane diisocyanate (pMDI) resin. ${ }^{9}$

In accordance with the environmental protection act of China, formaldehyde emissions must be considerably reduced to improve existing board methods in $2015 .{ }^{9}$ However, few

\footnotetext{
${ }^{a}$ Key Lab of Bio-based Material Science and Technology of Ministry of Education, Material Science and Engineering College, Northeast Forestry University, Harbin 150040, China. E-mail: gmh1964@126.com

${ }^{b}$ Heilongjiang Bayi Agricultural University, Daqing 163319, China

${ }^{c}$ Heilongjiang Provincial Key Laboratory of Environmental Microbiology and Recycling of Argo-Waste in Cold Region, College of Life Science and Technology, Heilongjiang Bayi Agricultural University, Daqing 163319, China

$\dagger$ These authors contributed equally to the work.
}

reports have depicted the development of formaldehyde-free panels, such as MDI. Importantly, although an alternative to formaldehyde, MDI exposure causes pulmonary diseases. A safe alternative adhesive is lignin adhesive,${ }^{\mathbf{1 5 - 1 8}}$ which is a new green formaldehyde-free adhesive ${ }^{\mathbf{1 9 - 2 1}}$ that can be employed in nonwood commercial production. Lignin oxidation with $\mathrm{H}_{2} \mathrm{O}_{2}$ may effectively improve the solvent safe utilization because water is used to decomposition, which eliminates the need for organic solvents and reducing environmental issues. Furthermore, alkaline aqueous solutions are excellent reaction media compared to acidic or neutral environments. ${ }^{22,23}$ Corn straw are industrial raw material sources that have numerous potential applications, including energy, materials, ${ }^{24}$ and chemical production. ${ }^{25}$ The usage of agricultural residues originating from the forest industry can minimize air pollution caused by the combustion of corn residues, and increase the sustainability, as well as effectively reduce costs in the artificial panel manufacturing industry. ${ }^{26}$ However, most known residues, such as stalks and husks, are burned or disposed owing to multiple limitations, including farming conditions and collection cost. ${ }^{\mathbf{1 4}}$

This paper investigates the feasibility of novel biocomposites via pretreatment of corn straw particles (PCSP) with a modified ammonium lignosulfonate (MAL) binder. Due to differences in polarity, corn straw requires pretreatment, but in order the make the procedure viable inexpensive reagents must be employed. In order to blend corn straw particles (CSP) and MAL homogeneously, polyethylenimine (PEI) is used efficiently to pretreat corn straw particles making them more 
compatible. However, little is known on whether PEI may improve the properties of CSP/MAL biocomposite. The purpose of this study is to demonstrate the effect of PEI pretreatment on characterization of the PCSP/MAL composites.

\section{Experimental}

\subsection{Materials}

Corn straw particles (moisture content: 5\%) were obtained from Anda (Heilongjiang Province, China) and passed through a 4060 mesh sieve for separation. The average chemical compositions of the initial particles were determined as $4.6 \%$ ash, $14.9 \%$ extractives, $16.7 \%$ lignin, $45.6 \%$ cellulose, and $22.5 \%$ hemicelluloses. ${ }^{10}$ Ammonium lignosulfonate (AL) was obtained from Shenyang Xingzhenghe Chemical Company (Shenyang, China) with the composition content determined as $51.9 \%$ total lignin, $27.1 \%$ carbohydrates, $10.6 \%$ ash, and $4.6 \%$ moisture. Polyethylenimine (PEI) was obtained from Shanghai UN Chemical (Shanghai, China). The molecular weight of PEI was 75000 in $50 \mathrm{wt} \%$ aqueous solution. All other chemicals were of analytical grade.

\subsection{Pretreatment of the corn straw particles (PCSP)}

$1 \mathrm{~kg}$ CSP of 40-60 mesh was placed into a closed blender, and $40 \mathrm{~g}$ PEI ( 2 wt\% PEI) and $10 \mathrm{~g}$ glutaraldehyde were mixed homogenously and sprayed into the blender. Then the mixture was stirred at $50{ }^{\circ} \mathrm{C}$ for $30 \mathrm{~min}$. They were then dried at $60^{\circ} \mathrm{C}$ for $12 \mathrm{~h}$ to stop the pretreatment process. The target moisture content of PCSP was set between $4 \%$ and $6 \%$.

\subsection{Preparation of modified ammonium lignosulfonate (MAL)}

MAL was prepared following a reported procedure: ${ }^{23} 1 \mathrm{~g}$ AL was dissolved in $10 \mathrm{~mL}$ distilled water and alkalinized adjusted to $\mathrm{pH}$ 10. Then, $10 \mathrm{~mL} \mathrm{H}_{2} \mathrm{O}_{2}$ was mixed with the AL solution, which was stirred at $60{ }^{\circ} \mathrm{C}$ for $30 \mathrm{~min}$. Thereafter, the mixture solution was concentrated to $20 \mathrm{wt} \%$ MAL solution.

\subsection{PCSP/MAL board preparation}

The mass ratio of PCSP to MAL was $85: 15$ according to previous reports. ${ }^{10}$ The blender particles were hand-filled into the mat of a $300 \mathrm{~mm} \times 300 \mathrm{~mm}$ forming box. The target density of each composite was determined as $0.8 \pm 0.03 \mathrm{~g} \mathrm{~cm}^{-3}$ with a target thickness of $5 \mathrm{~mm}$. Then the panels were stored under constant humidity at room temperature for 48 h. Fig. 1a and b show photographs of the prepared samples.

\subsection{Mechanical and dimensional properties}

The mechanical and dimensional properties of the composites were measured according to the Chinese National Standard GB/ $\mathrm{T} 17657$ (ref. 27) after conditioning at $20 \pm 2{ }^{\circ} \mathrm{C}$ and $65 \pm 5 \%$ relative humidity $(\mathrm{RH})$. According to the standard, the ratio of bending moment and modulus under the maximum load is defined as modulus of rupture (MOR). The ratio of stress and strain under the load in the elastic limit is defined as modulus of elasticity (MOE). The internal bonding strength (IB) is the ratio of the maximum damage tension perpendicular to the sample surface of the sample surface area. The sample $(200 \mathrm{~mm}$ $\times 50 \mathrm{~mm}$ ) was performed by three-point static bending to measure the MOR and MOE values at a loading speed of 5 $\mathrm{mm} \mathrm{min}^{-1}$. The sample $(50 \mathrm{~mm} \times 50 \mathrm{~mm})$ was pulled apart in the vertical direction to measure the IB value at a loading speed of $2 \mathrm{~mm} \mathrm{~min}^{-1}$. Fig. $1 \mathrm{c}$ and $\mathrm{d}$ show the test methods for the mechanical properties of the composites. Three samples of each target composite were measured for reproducibility.

The thickness swell (TS) and water absorption (WA) were measured by the percentage increase in thickness and weight of the sample after $24 \mathrm{~h}$ of immersion in water at room temperature. 8 specimens $(50 \mathrm{~mm} \times 50 \mathrm{~mm})$ of each target composite were analyzed. The surface hydrophobicity for each composite was measured at room temperature using JC2000A contact angle (CA). The load-bearing particleboard properties of GB/T 4897 (ref. 28) were determined as $\mathrm{MOR} \geq 15 \mathrm{MPa}, \mathrm{MOE} \geq$ $2200 \mathrm{MPa}, \mathrm{IB} \geq 0.45 \mathrm{MPa}$, and $24 \mathrm{~h}$ TS $\leq 22 \%$.

\subsection{X-ray diffraction analysis (XRD)}

The crystal structure and orientation of CSP and the prepared composites were studied using XRD with a wide angle. The $2 \theta$ angle ranges from $10^{\circ}$ to $45^{\circ}$ and set to $5^{\circ} \mathrm{min}^{-1}$ for the reflection mode scanning. The crystallinity index of the sample $(15 \mathrm{~mm} \times 15 \mathrm{~mm} \times 3.2 \mathrm{~mm})$ was calculated in accordance with previous reports. ${ }^{29}$

\subsection{Dynamic mechanical analysis (DMA)}

The storage modulus $\left(E^{\prime}\right)$ and $\tan \delta$ curves of the CSP/MAL and PCSP/MAL composites were analyzed utilizing a thermal mechanical instrument (DMA-242 model). The three-point bending mold was performed for the test. The samples $(50 \mathrm{~mm} \times 8 \mathrm{~mm} \times 5 \mathrm{~mm})$ were heated from 50 to $250{ }^{\circ} \mathrm{C}$ at a rate of $5{ }^{\circ} \mathrm{C} \min ^{-1}$.

\subsection{Scanning electron microscope (SEM)}

The micrographs of the CSP/MAL and PCSP/MAL composites were obtained using Sirion 200 (FEI, the Netherlands). Each sample was coated with a thin layer of gold, and electrically conductive by an ion sputtering coater. SEM images were performed at $12.5 \mathrm{kV}$ beam voltage.

\section{Results and discussion}

\subsection{Effect of PEI dosage on the mechanical properties}

The effect of PEI dosage on the mechanical properties is shown in Fig. 2. The straight line parallel to the $x$-axis manifests the minimum requirements of Chinese national standard. ${ }^{28}$

The effects of PEI dosage on MOR and MOE of the CSP/MAL composites are shown in Fig. 2a. MOR increases significantly with increasing binder content from 0 to $2 \mathrm{wt} \%$. In the case of 2 to $2.5 \mathrm{wt} \%$, a slight decrease in MOR occurs. However, increasing dosage from 2.5 to $3.0 \mathrm{wt} \%$, gave no significant change in MOR. With increasing PEI dosage from 0 to $2.0 \mathrm{wt} \%$, an intense increase in MOE is observed, whereas PEI dosage 
from 2.0 to $3.0 \mathrm{wt} \%$ did not display any such changes in MOE. When PEI dosage is greater than $1.0 \mathrm{wt} \%$, MOR and MOE values exceed the minimum requirements (horizontal dashed and solid lines). Fig. 2b shows the effect of PEI dosage on IB of the CSP/MAL composites. There are two stages in the IB trend. Firstly, PEI dosage is in the range of $0-2 \mathrm{wt} \%$, but with increases with increasing IB value. It is indicative of CSP crosslinking with PEI to improve bonding strength. In second stage of 2 to $2.5 \mathrm{wt} \%$, a dramatic decrease in IB occurs. These results suggested that a high amount of PEI could lead to deterioration of mechanical performance of PCSP/MAL composites.

When PEI dosage is greater than $1.5 \mathrm{wt} \%$, the IB values exceed minimum requirement (horizontal dashed line). By comparing $0 \% \mathrm{PF}$ with $2 \% \mathrm{PF}$, the IB value increased eight fold, escalating from below 0.1 MPa to more than 0.4 MPa. After PEI pretreatment, MOR and MOE show an overwhelming increase. These significant changes in mechanical properties, especially IB, indicate that the interphase between MAL and CSP has been improved. The best values were obtained for $2 \%$ PEI: an MOR of 32.29 MPa, an MOE of 4001.15 MPa, and an IB of 1.17 MPa were measured, corresponding to an increase of approx. 996.76\%, $712.14 \%$ and $775.00 \%$, respectively, compared to the CSP/MAL composites without PEI pretreatment. Moreover, according to the above results, it's feasible to use MAL as adhesive instead of formaldehyde resin.

\subsection{Effect of PEI dosage on the dimensional properties}

The effects of PEI dosage on the TS and WA values of the composites are shown in Fig. 3. In general, PEI-treated CS bio-
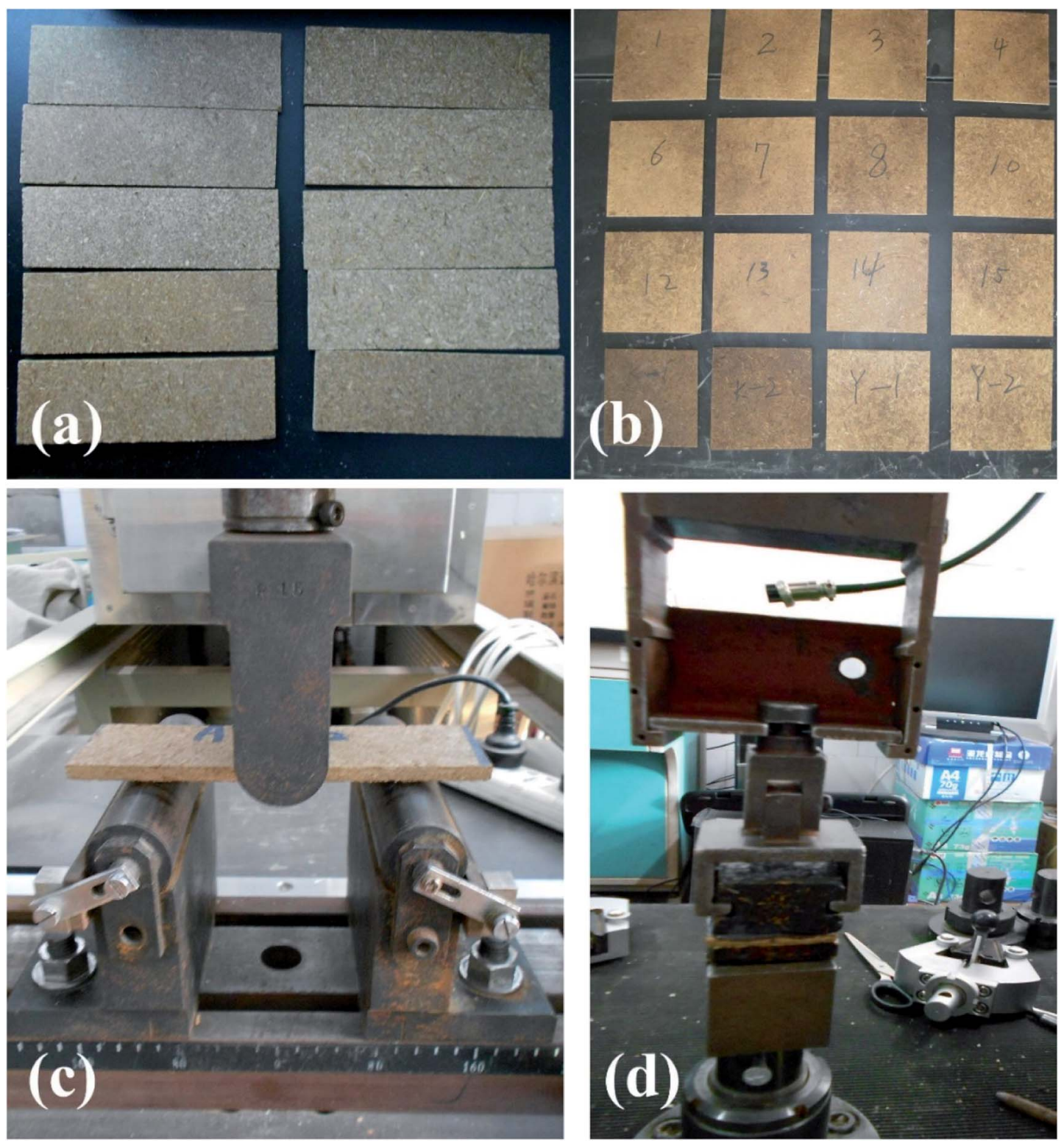

Fig. 1 Photographs of the fabricated bio-composites: (a) samples of MOR and MOE with the dimensions of $200 \mathrm{~mm} \times 50 \mathrm{~mm} \times 5 \mathrm{~mm}$, (b) samples of IB with the dimensions of $50 \mathrm{~mm} \times 50 \mathrm{~mm} \times 5 \mathrm{~mm}$; (c) test mode of MOR and MOE; (d) test mode for IB. 

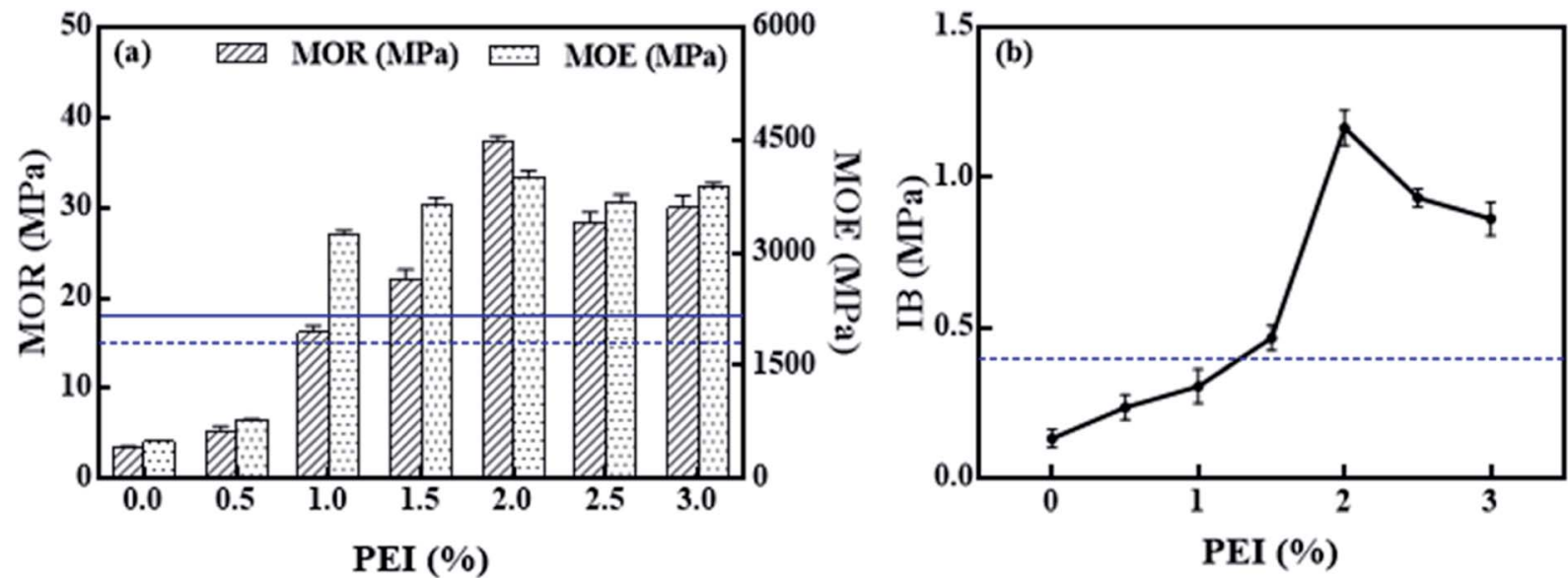

Fig. 2 Effect of PEl treatment on the mechanical properties of corn straw bio-composites: (a) MOR and MOE, (b) IB.

board exhibits low TS and WA. When PEI dosage increases from 1.0 to $2.5 \mathrm{wt} \%$, TS value decreases significantly, however, from 2.5-3.0 wt\% TS value shows a remarkable increase. Improvements in the dimensional properties of biocomposites could be attributed to the hydrophobicity substances from MAL reacting with PEI of CSP surface, which prevented water from entering into its molecular chains. ${ }^{23}$ The TS must be lower than $22 \%$ for load-bearing particleboard. ${ }^{28}$ Furthermore, at 2.0 wt $\%$ and $2.5 \mathrm{wt} \%$ PEI dosage, the TS values meet minimum requirement (horizontal dashed line).

The effect of PEI dosage on CA of the CSP bio-board with $15 \mathrm{wt} \%$ MAL is shown in Fig. 4. With increasing PEI dosage, the CA trend displays three distinct stages. Firstly, when PEI dosage is from 0 to $0.5 \mathrm{wt} \%, \mathrm{CA}$ is approx. $90^{\circ}$ to $25^{\circ}$ after $30 \mathrm{~s}$. The final CA is measured at approx. $8^{\circ}$ after $60 \mathrm{~s}$, which means very poor water resistance. Secondly, PEI dosage ranging between 1 and $1.5 \mathrm{wt} \%$ and $3 \mathrm{wt} \%$, shows a change from $100^{\circ}$ to $40^{\circ}$ within $60 \mathrm{~s}$, which also implies that the composite is hydrophilic. Furthermore, at dosage between 2 and $2.5 \mathrm{wt} \%$, the CA is greater than $70^{\circ}$ after $60 \mathrm{~s}$, which indicates favourable hydrophobicity. Hence, the PEI dosage has a positive effect on the water resistance of the composites. This shows that poor

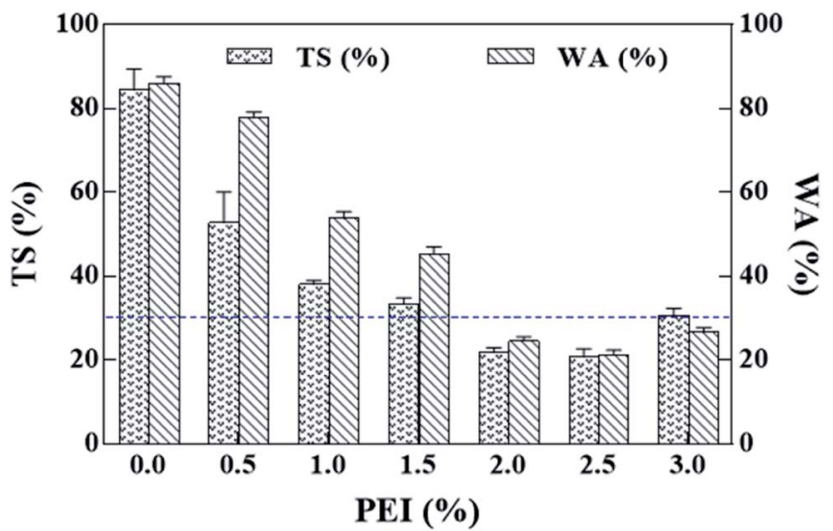

Fig. 3 Effect of PEl treatment on the dimensional stability of corn straw bio-composites.

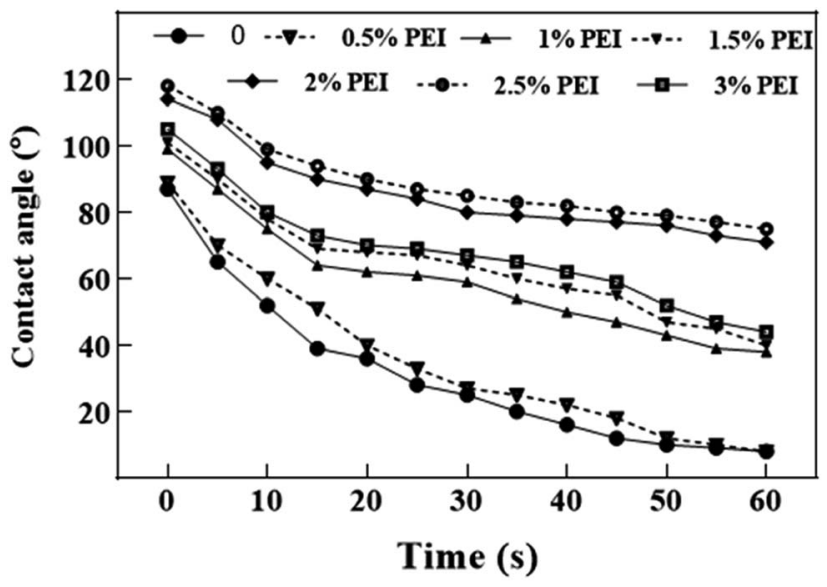

Fig. 4 Effect of PEl dosage on CA at the surface of the composites.

interfacial adhesion makes the combination of MAL and CSP with too high or too low PEI not strong enough, and there maybe crakes, which leads to the infusion of water and

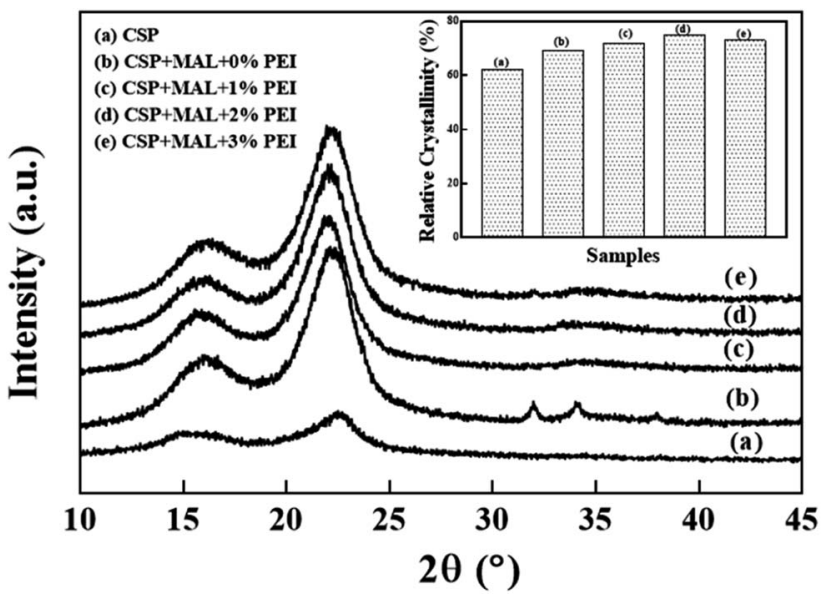

Fig. 5 X-ray diffraction patterns obtained for the CSP, CSP/MAL and PCSP/MAL composites. 


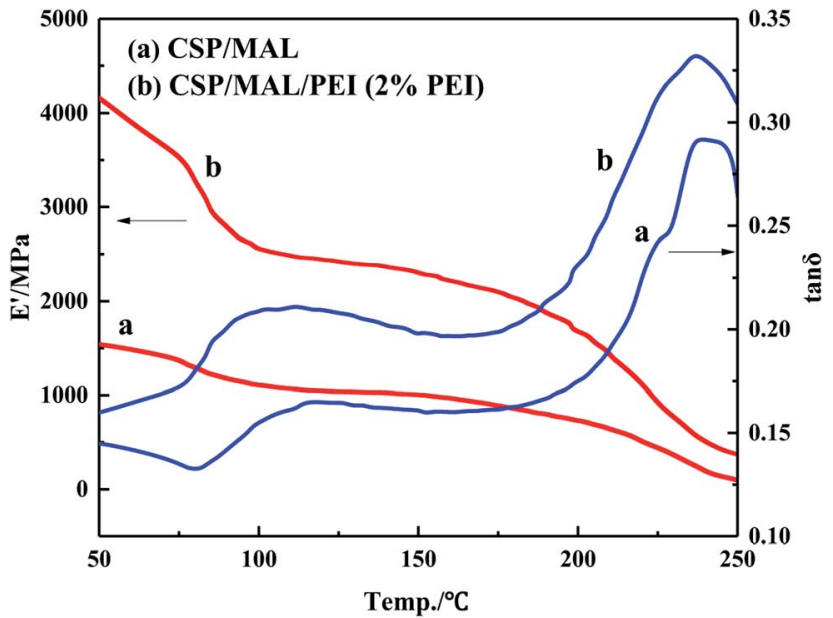

Fig. 6 The storage modulus $E^{\prime}$ and $\tan \delta$ curves of CSP/MAL (a) and PCSP/MAL composites (b).

subsequent fiber swelling. The suitable water resistance of the PCSP/MAL composites is $2-2.5 \mathrm{wt} \%$ of PEI dosage.

Before curing, the amine groups of PEI was grafted onto many -OH groups of CSP with the cross-linking of glutaraldehyde, meaning a high compatibility. Therefore, PEI can adhere well to the CSP surface. After curing, PEI of the corn straw surface could react with quinones of MAL enhancing strength and achieve good water-resistant. The binder mechanism is similar to the quinone-tannin processes in nature, ${ }^{30}$ where various reactions between the amino group and the catechol group solidify and crosslink the marine adhesive protein with strong and very water-resistant property. ${ }^{31}$ Hence active groups in MAL, such as phenolic hydroxyl and aldehyde groups, react with PCSP to form bonds. ${ }^{32}$ Hence, PEI significantly improves the interface properties of MAL and CSP, as well as their mechanical properties and dimensional stability.

\subsection{X-ray diffraction analysis}

Fig. 5 shows the crystallinities of the CSP composites with different PEI content. Diffraction peaks of all samples are found at $2 \theta=18.2^{\circ}$ and $22.1^{\circ}$ and are in accord with the typical cellulose I pattern. ${ }^{33}$ Therefore, the original crystal integrity of CSP cellulose is almost completely maintained during preparation. The diffraction peak of the sample is similar to that of natural cellulose, but differs from microwave-assisted,$^{34} \mathrm{chem}-$ ical $^{35}$ and biological treatment, ${ }^{36}$ which changed the crystal integrity of cellulose.

The apparent crystallinity of the CSP is $61.96 \%$, but after manufacturing with MAL, major alterations to the composites crystallinity occurs. In the absence of PEI pretreatment, the crystallinity of the CSP/MAL composite increases to $69.23 \%$. At $1 \mathrm{wt} \%$ and $2 \mathrm{wt} \%$ PEI, the apparent crystallinity of the PCSP/ MAL composite is further increased to $71.69 \%$ and $74.77 \%$, respectively. This indicates that PEI possesses good surface activity while having a positive effect on the CSP composite
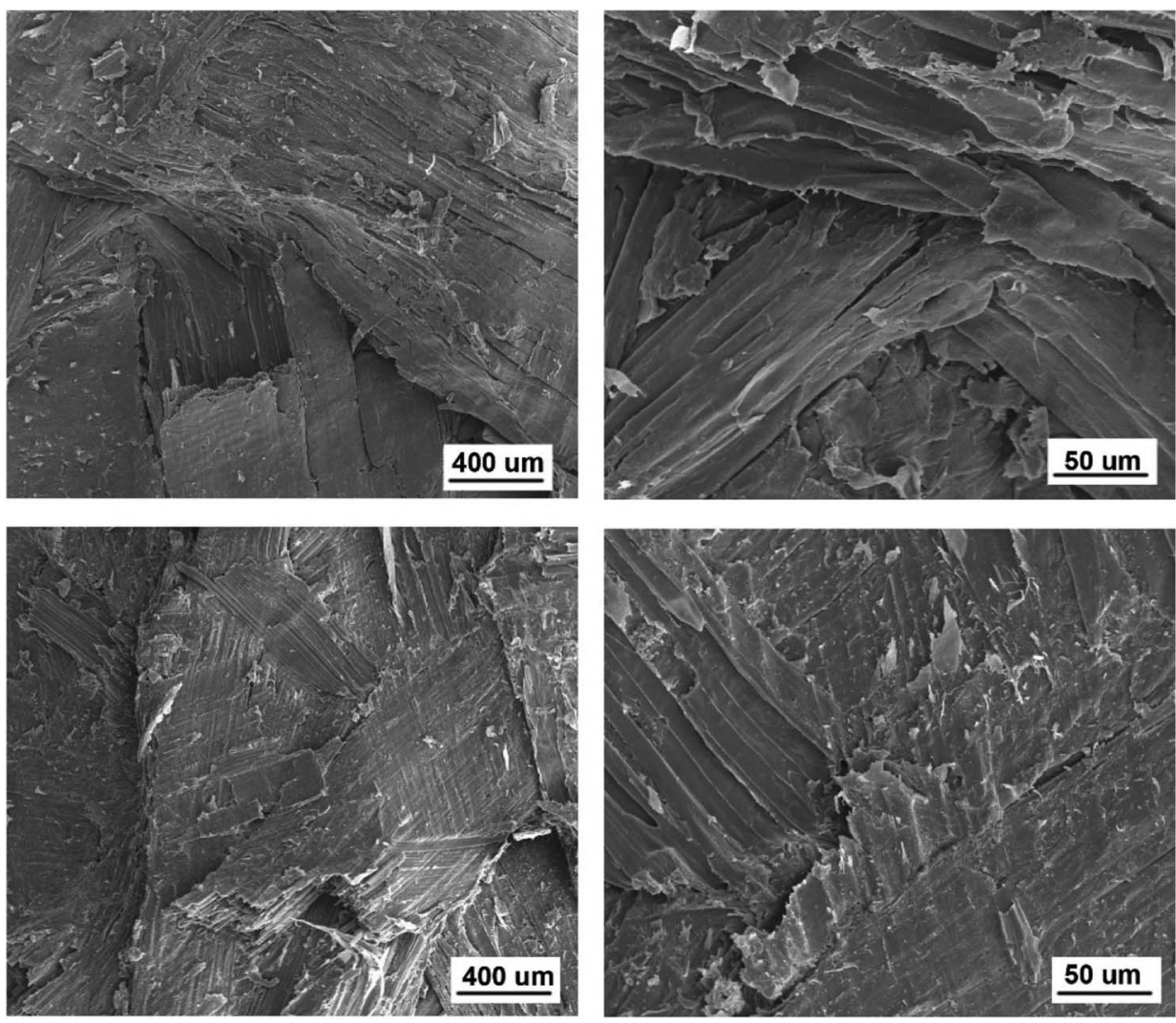

Fig. 7 SEM images of the CSP/MAL ( $a$ and $b$ ) and PCSP/MAL composites (c and d). 
crystallinity. Biopolymers such as cellulose and MAL contain a random array of crystalline and amorphous phases. However, introduction of PEI into the matrix may accelerate the nucleation step or lamellar rearrangement of the crystallization reaction of cellulose and MAL in the crystallization process, forming a small number of thinner crystals adhering to the original crystallites. Therefore, when PEI dosage is increased to $3 \mathrm{wt} \%$, the crystallinity of CSP/MAL/PEI composite displays a slight decrease $(72.87 \%)$. This shows that at higher PEI dosage adverse effects occur in crystallization of the PCSP/MAL composites.

\subsection{Dynamic mechanical analysis (DMA)}

DMTA was employed to demonstrate the viscoelastic properties of the composites. ${ }^{37,38}$ The storage modulus $E^{\prime}$ and tan $\delta$ curves of CSP/MAL and PCSP/MAL composites are shown in Fig. $6 . E^{\prime}$ of $\mathrm{PCSP} / \mathrm{MAL}$ composites is markedly higher than that of CSP/MAL composites. The maximum modulus of PCSP/MAL composites is $4159.76 \mathrm{MPa}$. The obtained results are consistent with the crystallinity data, as shown above in Fig. 5. The plots of $\tan \delta$ value versus temperature are measured for the segmental motion of polymer molecules. Thus, the peak of $\tan \delta$ is explained as $\mathrm{Tg}$. The tan $\delta$ peaks for the composites are $237^{\circ} \mathrm{C}$. Hence, PEI pretreatment has no obvious influence on $\mathrm{Tg}$. Moreover, the $\tan \delta$ value of PCSP/MAL composites is 0.33 which appears higher than that of CSP/MAL composites, which suggests that PEI pretreatment influences the mobility of CSP chains during board manufacturing. ${ }^{39}$

\subsection{Morphology of particles and composites}

Fig. 7 shows the micrographs of the CSP/MAL and PCSP/MAL composites at low and high magnifications, respectively. Without PEI pretreatment, the corn straw fiber cell walls almost retain their original morphology at high press temperatures.

Fig. 7a and $\mathrm{b}$ show that the CSP/MAL composites possess more voids (directed by the arrows). Due to CSP/MAL composites poor adhesion, the fibers are obviously debonded. Employing $2.0 \mathrm{wt} \%$ PEI pretreatment, the void ratio of PCSP/ MAL composites is significantly reduced, which improves the compression properties of corn straw fibers, as shown in Fig. 7c and $d$. During the process of composite formation, the presence of PEI promotes the combination of the so-called glue line. ${ }^{31}$ Therefore, CSP treated by PEI strongly interacts with MAL molecules, this interaction is weaker when PEI treatment is not employed. This proves that PEI pretreatment improves the interfacial adhesion between MAL and CSP particles.

\section{Conclusions}

For the first time, new bio-composites were prepared using PCSP and MAL binders. In order to improve the interfacial adhesion between MAL and CSP, novel PEI treatment of CPS was adopted. The obtained results determined PEI pretreatment markedly improved the interfacial compatibility of the PCSP/MAL composites. Due to the rein-forcing effect of PEI surface treatment on the matrix, the PCSP/MAL composites showed an increase of 52.94-996.76\% for MOR, 58.46-684.84\% for MOE and $77.50-775.00 \%$ for IB compared with CSP/MAL composites (85/15). The optimum MOR, MOE, IB, TS and WA of the PCSP/MAL composites complied with the requirement of load-bearing particleboard, which were $37.29 \mathrm{MPa}$, 4001.15 MPa, 1.22 MPa, 21.77\% and 24.52\%, respectively. However, PEI negatively affected the mechanical properties and dimensional stability of the PCSP/MAL composites above 2.5 wt\% PEI dosage. Furthermore, PEI displayed good surface activity and plays a positive role in improving the crystallinity of PCSP/MAL composites. This method may enable a new and lowcost method for the modification of straw particles and manufacturing of formaldehyde-free particleboards. These renewable and environmentally friendly composites may be suitable for commercial production. Further studies in this area will systematically focus on the bonding mechanisms of the interface.

\section{Conflicts of interest}

There are no conflicts to declare.

\section{Acknowledgements}

This work was supported by the Special Fund for Forest Scientific Research in the Public Welfare (Grant No. 201504501-1); the National Natural Science Foundation of China (Grant No. 31801313); University Nursing Program for Young Scholars with Creative Talents in Heilongjiang Province (Grant No. UNPYSCT2018085); Open Foundation of the Heilongjiang Provincial Key Laboratory of Environmental Microbiology and Recycling of Argo-Waste in Cold Region (Grant No. 201708); and the Doctor Initial Foundation of Heilongjiang Bayi Agricultural University (Grant No. XYB 2015-10).

\section{References}

1 D. H. Wang and X. S. Sun, Ind. Crops Prod., 2002, 15, 43-50.

2 R. Jarabo, M. C. Monte, E. Fuente, S. F. Santos and C. Negro, Ind. Crops Prod., 2013, 43, 832-839.

3 J. G. Wu, X. Zhang, J. L. Wan, F. Y. Ma, Y. Tang and X. Y. Zhang, Bioresour. Technol., 2011, 102, 11258-11261.

4 I. Ali, K. Jayaraman and D. Bhattacharyya, Ind. Crops Prod., 2015, 67, 422-431.

5 J. Y. Xu, G. P. Han, E. D. Wong and S. Kawai, J. Wood Sci., 2003, 49, 327-332.

6 J. Y. Xu, R. Sugawara, R. Widyorini, G. P. Han and S. Kawai, J. Wood Sci., 2004, 50, 62-67.

7 X. J. Li, Z. Cai, J. E. Winandy and A. H. Basta, Bioresour. Technol., 2010, 101, 4662-4666.

8 X. Y. Zhou, F. Zheng, H. G. Li and C. L. Lu, Energy and Buildings, 2010, 42, 1070-1074.

9 R. Li, C. Lan, Z. Wu, T. Huang, X. Chen, Y. Liao, L. Ye, X. Lin, Y. Yang and Y. Zheng, Constr. Build. Mater., 2017, 148, 781788.

10 Y. Yuan, L. Sidan, J. Feng, S. Guinan, Y. Lei and W. Weidong, BioResources, 2019, 14, 5923-5942. 
11 S. Halvarsson, H. Edlund and M. Norgren, Ind. Crops Prod., 2008, 28, 37-46.

12 Y. Zhang, Z. B. He, L. Xue, D. M. Chu and J. Mu, RSC Adv., 2016, 6, 12850-12861.

13 G. A. Holt, P. Chow, J. D. Wanjura, M. G. Pelletier and T. C. Wedegaertner, Ind. Crops Prod., 2014, 52, 627-632.

14 Y. Çöpür, C. Güler, M. Akgül and C. Taşçığlu, Building and Environment, 2007, 42, 2568-2572.

15 X. Ji, Y. Dong, B. Yuan, B. Li and M. Guo, J. Appl. Polym. Sci., 2018, 135, 45870.

16 X. Ji and M. Guo, Int. J. Adhes. Adhes., 2018, 82, 8-13.

17 J. A. Velásquez, F. Ferrando and J. Salvadó, Ind. Crops Prod., 2003, 18, 17-23.

18 V. Hemmilä, S. Adamopoulos, O. Karlsson and A. Kumar, RSC Adv., 2017, 7, 38604-38630.

19 Y. Liu and K. C. Li, J. Adhes., 2006, 82, 593-605.

20 N. E. Mansouri, A. Pizzi and J. Salvadó, Holz Roh-Werkst., 2007, 65(66), 65-70.

21 Y. Yuan and M. Guo, Int. J. Life Cycle Assess., 2017, 22, 13181326.

22 J. P. Hu and M. H. Guo, Ind. Crops Prod., 2015, 78, 48-57.

23 Y. Yuan, M. H. Guo and F. Y. Liu, BioResources, 2014, 9, 836848.

24 A. Wu, J. Yan, W. Xu and X. Li, Mater. Lett., 2016, 173, 60-63. 25 O. Ioannidou, A. Zabaniotou, E. V. Antonakou, K. M. Papazisi, A. A. Lappas and C. Athanassiou, Renewable Sustainable Energy Rev., 2009, 13, 750-762.
26 C. Juárez, A. Durán, P. Valdez and G. Fajardo, Building and Environment, 2007, 42, 1151-1157.

$27 \mathrm{~GB} / T$ 17657, Standardization Administration of China, Beijing, China, 2013.

$28 \mathrm{~GB} / \mathrm{T}$ 4897, Standardization Administration of China, Beijing, China, 2015.

29 L. Segal, J. J. Creely, A. E. Martin and C. M. Conrad, Text. Res. J., 1959, 29, 786-794.

30 X. Geng and K. Li, J. Adhes. Sci. Technol., 2006, 20, 847-858. 31 Y. Liu and K. C. Li, J. Adhes., 2006, 82, 593-605.

32 J. P. Hu and M. H. Guo, Sci. Silvae Sin., 2013, 49, 103-109.

33 Y. Nishiyama, J. Sugiyama, H. Chanzy and P. Langan, J. Am. Chem. Soc., 2003, 125, 14300-14306.

34 F. H. Li, H. J. Hu, R. S. Yao, H. Wang and M. M. Li, Ind. Eng. Chem. Res., 2012, 51, 6270-6274.

35 M. Martelli-Tosi, O. B. G. Assis, N. C. Silva, B. S. Esposto, M. A. Martins and D. R. Tapia-Blácido, Carbohydr. Polym., 2017, 157, 512-520.

36 J. J. Zeng, D. Singh and S. L. Chen, Bioresour. Technol., 2011, 102, 3206-3214.

37 P. C. Rodrigues, M. Muraro, C. M. Garcia, G. P. Souza, M. Abbate, W. H. Schreiner and M. A. B. Gomes, Eur. Polym. J., 2001, 37, 2217-2223.

38 K. Joseph, S. Thomas and C. Pavithran, Mater. Lett., 1992, 15, 224-228.

39 A. Kaushik, M. Singh and G. Verma, Carbohydr. Polym., 2010, 82, 337-345. 